\title{
BMJ Open Study protocol: a pilot randomised waitlist-controlled trial of a dyadic mobile health intervention for black sexual-minority male couples with HIV in the USA
}

Hyunjin Cindy Kim (D) , Lance M Pollack, Parya Saberi (1) , Torsten B Neilands, Emily A Arnold, Darius Jovon Bright, Robert W Williams, Susan M Kegeles, Judy Y Tan

To cite: Kim HC, Pollack LM, Saberi P, et al. Study protocol: a pilot randomised waitlist-controlled trial of a dyadic mobile health intervention for black sexualminority male couples with HIV in the USA. BMJ Open 2021;11:e055448. doi:10.1136/ bmjopen-2021-055448

- Prepublication history for this paper is available online. To view these files, please visit the journal online (http://dx.doi. org/10.1136/bmjopen-2021055448).

Received 14 July 2021 Accepted 17 August 2021

Check for updates

(C) Author(s) (or their employer(s)) 2021. Re-use permitted under CC BY-NC. No commercial re-use. See rights and permissions. Published by BMJ.

Division of Prevention Science, Department of Medicine, University of California, San Francisco, San Francisco, California, USA

Correspondence to Hyunjin Cindy Kim; hyunjin.kim2@ucsf.edu

\section{ABSTRACT}

Introduction HIV care engagement is lower among black sexual-minority men relative to other racial/ethnic groups of sexual-minority men. Being in a primary relationship is generally associated with more successful HIV care engagement across various populations. However, among black sexual-minority men, the association between primary relationship status and HIV-related outcomes is inconsistent across the HIV care continuum. Given the ubiquity of mobile technology access and use among racial/ethnic minority communities, leveraging mobile technology for HIV care engagement appears a promising intervention strategy. This paper outlines the protocol of the LetSync study, a pilot randomised controlled trial of a mobile health app intervention developed using the Framework of Dyadic HIV Care Engagement to improve care-engagement outcomes among black sexual-minority male couples living with HIV.

Methods and analysis Eighty black sexual-minority men in couples $(n=160)$ will be enrolled to pilot test the LetSync app. At least one member of each dyad must be both HIV-positive and self-identify as black/African-American. Couples will be randomised to either a waitlist-control arm or an intervention that uses relationship-based approach to improve HIV care engagement. We will assess feasibility and acceptability of trial procedures and intervention protocols based on predefined metrics of feasibility and acceptability. Execution of the study will yield the opportunity to conduct analyses to test the measurement and analysis protocol on antiretroviral therapy adherence by comparing the intervention and waitlist-control arms on self-reported and biological (hair sample) measures of adherence.

Ethics and dissemination Study staff will obtain electronic consent from all participants. This study has been approved by the University of California (UCSF) Institutional Review Board. Study staff will work with the Community Advisory Board at the UCSF Center for AIDS Prevention Studies Board to disseminate results to participants and the community via open discussions, presentations, journal publications and/or social media. Trial registration number NCT04951544.
Strengths and limitations of this study

- Mobile health interventions have traditionally focused on a single user's experience and outcomes, which LetSync will challenge by harnessing couples' resilience and ability to problem-solve together, both of which impact dyadic coordination and, in return, can improve HIV care engagement.

- Involving participants in the app development process can allow for higher chance of acceptability of future iterations.

- The remote nature of our study breaks down barriers to participation such as travel, time and expenses.

- This intervention does not allow users to directly engage with the healthcare system.

- Due to this being a couples' study, it is possible that couples can break up during study participation which can impact feasibility results of the app.

\section{INTRODUCTION}

Black sexual-minority men (ie, gay, bisexual and other men who have sex with men (MSM)) account for $26 \%$ of 37968 new HIV diagnoses in the USA in 2018 and $37 \%$ of new diagnoses among all MSM. ${ }^{12}$ Black MSM also show the least favourable HIV care engagement outcomes (ie, testing, linkage to and retention in HIV care, viral suppression) relative to other $\mathrm{racial} / \mathrm{ethnic}$ groups of MSM. $^{34}$ Suboptimal adherence to antiretroviral therapy (ART) can lead to transmission and detrimental clinical outcomes. ${ }^{56}$ Based on current data, it is estimated that one in two black MSM will be diagnosed with HIV during their lifetime. ${ }^{78}$

National estimates show that a third to a half of black MSM with HIV are in a primary relationship, ${ }^{9-11}$ which is associated with favourable outcomes in healthcare engagement 
via social support pathways. ${ }^{12-15}$ Dyadic approaches are part of a multilevel intervention approach; yet, they remain poorly understood among black MSM. ${ }^{16}$ Emergent evidence show that black MSM in couples help each other engage in HIV care and treatment but that many do so inconsistently. ${ }^{17}{ }^{18}$ Additional characteristics of the dyad may moderate the effect of a primary relationship on HIV care engagement. ${ }^{14}{ }^{15}{ }^{19-21}$ For example, black couples with HIV may engage in joint problem-solving, a collaborative problem-focused approach to coping with stress, and dyadic coordination, or the synchronisation of activities and behaviours necessary in HIV care and treatment. ${ }^{1822}$

With $>75 \%$ of the US adult population owning smartphones, ${ }^{23}$ mobile health (mHealth) has emerged as a promising tool in healthcare including HIV prevention, care, and management efforts. ${ }^{24-26}$ Although mHealth has been shown to be feasible, acceptable and effective among black MSM, ${ }^{26-35}$ no dyadic mHealth interventions exist for this population even as black MSM face many unique barriers to care and treatment. ${ }^{36}$ Compared with white MSM, black MSM are 20\% less likely to be linked to, engaged and retained in HIV care due to social and structural inequities such as racial discrimination, ${ }^{37}$ access to ART, ${ }^{38}$ food and housing insecurity ${ }^{40} 41$ and overcriminalisation and policing of black communities. ${ }^{39}$ Low retention rates can also be explained by inequities in the healthcare system, such as experiencing stigma and shame from healthcare providers. ${ }^{42}$ Black sexual-minority couples show great interest in using a couples-based app to facilitate joint problem-solving to coordinate care and treatment activities, and provided ideas for the app features they want. ${ }^{22}{ }^{36}$ In contexts where same-sex relationships are highly stigmatised, black sexual-minority couples may appreciate an app that focuses on their primary romantic relationships.

Guided by the Framework of Dyadic HIV Care Engagement (figure 1), ${ }^{18} 22$ initial designs were created for a dyadic mHealth application (app) intervention called LetSync, for let's synchronise', to target dyadic

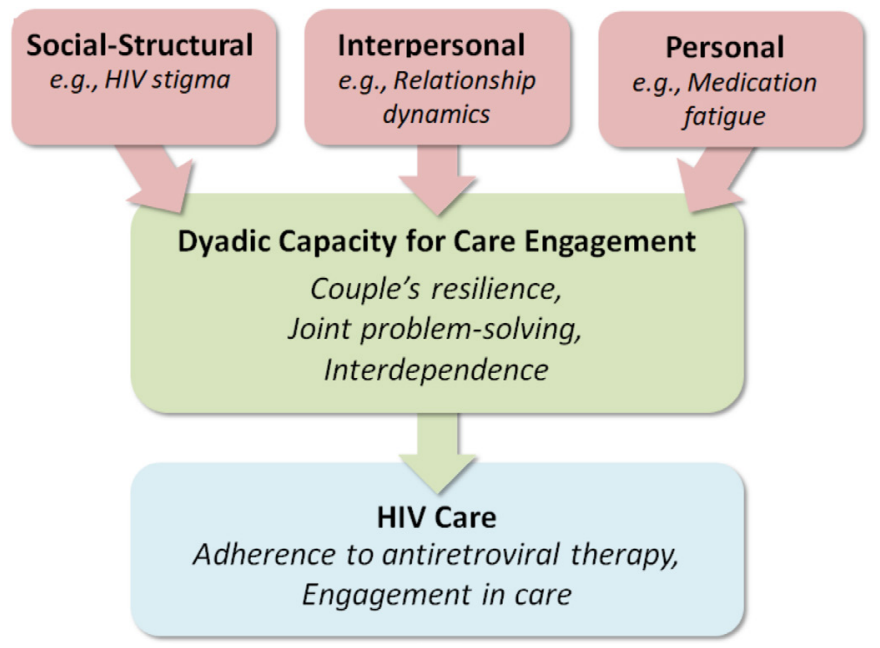

Figure 1 Framework of Dyadic HIV Care Engagement. coordination and joint problem-solving skills to improve retention in care and ART adherence. LetSync aims to facilitate among couples the dyadic coordination and joint problem-solving necessary for optimal engagement in HIV care among black MSM.

This protocol paper describes the pilot randomised waitlist-controlled trial to assess the feasibility and acceptability of the study protocols and procedures, assess the feasibility and acceptability of using LetSync and test measurement and analysis protocols on preliminary data of app use on ART adherence. Fully developing a couplesbased mHealth intervention will require that we translate findings to inform LetSync designs and iteratively develop, refine and pilot-test prototypes for a large-scale, future efficacy trial.

\section{METHODS AND ANALYSIS}

\section{Setting and participants}

LetSync is a single-site, pilot randomised waitlistcontrolled trial with the primary goal of assessing feasibility and acceptability of the mobile app, LetSync, among 80 black sexual-minority couples $(n=160)$ living in the USA. The sample size was chosen to be adequate to gauge feasibility and acceptability while remaining feasible for a pilot. Participants will be randomised to immediately begin the intervention or wait 6 months. A waitlist-control design (figure 2) will allow us to evaluate two versions of LetSync, a later version iteratively refined based on feedback about the previous version. ${ }^{43}$ LetSync will be developed by a third-party app developer to be compatible with both iOS and Android.

Participation in the study will last 14 months, with assessments conducted at baseline, 6, 8 and 14 months. We will collect feasibility and acceptability data, as well as preliminary data on ART adherence as measured by antiretroviral (ARV) concentrations in hair. Participants will consent to the study and complete an initial baseline survey online. Study staff will communicate with participants through text, email, phone and Zoom. The University of California, San Francisco (UCSF) Institutional Review Board (IRB) has reviewed and approved this study.

\section{Eligibility}

Black MSM who are at least 18 years of age, living with HIV in the USA and in a primary relationship with another man for at least 2 months will be eligible to participate. A primary relationship will be defined as a commitment to someone over and above anyone else that has lasted at least 3 months and includes a sexual relationship. ${ }^{44}$

At least one member of the couple must be AfricanAmerican/black and living with HIV (Index) who is either not on ART or is $<100 \%$ ART adherent as assessed via a three-item adherence measure. ${ }^{45}$ Their primary partner can be of any race or ethnicity, and any HIV status. Among couples where both partners meet eligibility as an Index, one will be chosen at random to be the 


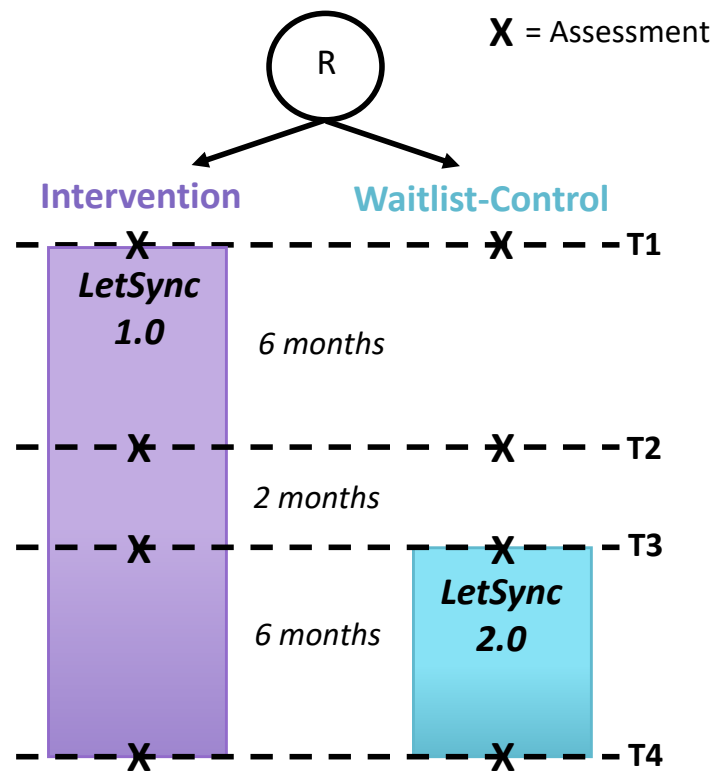

Figure 2 Timeline of LetSync intervention.

Index. Both members of the couple must own or have access to a smartphone.

We will exclude individuals who (1) report fear of intimate partner violence resulting from participation as assessed at screening, ${ }^{46} 47$ (2) are unwilling or unable to disclose HIV status to primary partner or (3) are presenting evidence of severe cognitive impairment that would prevent comprehension of study procedures assessed during informed consent.

\section{Patient and public involvement}

Prior to the design of LetSync, investigators conducted formative research with black sexual-minority men in the San Francisco Bay Area. Black sexual-minority couples showed strong mHealth preferences and interest in using a mobile app to facilitate joint problem-solving to achieve optimal HIV care engagement. ${ }^{36}$ We will also assemble a Community Advisory Board of black sexual-minority couples to obtain feedback on LetSync prototypes and develop LetSync V.1.0.

\section{Study procedures}

\section{Recruitment}

We will use a multipronged recruitment approach that includes in-person and virtual engagement. In addition to the San Francisco Bay Area, we will prioritise recruiting from US cities with the highest prevalence of HIV among black MSM (eg, Atlanta, Georgia; Los Angeles, California; Washington, District of Columbia; Houston, Texas). We will attend virtual events hosted by community-based organisations serving black/African-American and/or sexual-minority communities impacted by HIV/AIDS, placing targeted online advertisements on social media (eg, Facebook) and asking clinics that serve black MSM with HIV to distribute flyers. We will also recruit from within UCSF clinics via the UCSF Recruitment Letter Services. We will also contact participants of other UCSF studies who gave consent to be contacted.

\section{Screening}

Study staff will provide a brief overview of the study to prospective participants, answer any questions and complete an eligibility screening over the telephone. Targeted online advertisements will link to an online prescreener that interested individuals can take to see if they qualify. Only those who are potentially eligible (based on screener responses) will be contacted by study staff. Ineligible responses will be recorded along with the reasons why (eg, not living with HIV, not in relationship with a man).

\section{Consent/Enrolment}

If found to be eligible on screening, individuals will be sent an informed consent form online. Eligible individuals will be instructed to read the consent form in full and ask any questions they may have prior to giving consent. Study staff will be available to respond to any questions or concerns and to ensure comprehension. 


\section{Intervention}

\section{Randomisation}

After obtaining informed consent from both members of the dyad, we will randomise couples to the intervention or waitlist-control groups using a randomisation-plan generator.

\section{Intervention content: LetSync}

To enhance the couples' capacity for HIV care engagement, LetSync was designed based on problem-solving therapy. Problem-solving therapy consists of distinct steps to help identify problems one may have, possible solutions to follow and the advantages and disadvantages to each. ${ }^{48}$ Problem-solving therapy has shown to be effective in other mHealth interventions (eg, iProblemSolve, a goal-setting app targeting individuals). ${ }^{49}$

The defining feature of LetSync is 'My Action Plan', which will guide the Index to arrive at a tailored action plan that addresses a component of HIV care engagement. The Index will identify current HIV care engagement and general health-related issues, choose strategies for addressing the issues (strategies already extant in the app plus new strategies the user can add) and evaluate those strategies in terms of likelihood of implementation. The Action Plan, which is composed of the strategies the user identified as most likely to be implemented, can then be shared with their partner through the app. The Action Plan will contain features to encourage the Index and their partner to engage in joint problem-solving and dyadic coordination. For example, partners will be prompted to make suggestions to Action Plans, download the Action Plans into their own mobile calendars, view goals and progress, coordinate activities around goals and appointments and share encouragements.

\section{Timeline}

The study timeline will be split into four time points $(\mathrm{T})$ : T1 (baseline), T2 (6 months), T3 (8 months) and T4 (14 months) (figure 2).

At $\mathrm{T} 1$, participants in the intervention and waitlistcontrol arms will receive hair-sample collection kits in the mail with necessary supplies, an electronic link to an instructional video and a pre-paid envelope for returning samples. ${ }^{50}$ Participants in the intervention arm will receive an electronic link to the baseline survey and will be scheduled their first study visit, which will occur via videoconference (eg, Zoom). At the first study visit, study staff will give an overview of the study, answer any questions and assist the participant in installing the app on their phone and provide necessary instructions for app use. The intervention group will use LetSync V.1.0 for 6 months.

At all three subsequent time points (T2-T4), participants in both arms will receive a text or email informing them that the next study assessment is due, along with the link to complete the assessment. Simultaneously, we will mail all participants a hair-sample kit.
Between T1 and T2, we will collect data on acceptability and feasibility and use this to revise LetSync V.1.0 and update it to LetSync V.2.0.

At T3, participants in the waitlist-control arm will attend a videoconference during which study staff will offer an overview of the study, answer any questions and assist the participant in installing and using LetSync V.2.0. Meanwhile, the participants in the intervention arm will continue to use LetSync V.1.0.

At T4, we will conduct virtual exit interviews with participants from both arms over the phone or via videoconference. During exit interviews, we will ask for feedback about the randomisation procedures to inform future randomised controlled trial (RCT) procedures. Interviews will be audio-recorded for transcription and data analyses.

\section{Incentives}

Participants will receive a US $\$ 50$ cash card, payment through a cash app or reloadable debit card on completing each survey, an additional US $\$ 50$ on receipt of hair samples at T1, T2, T3 and T4, and US\$30 for completing the exit interview at T4. Altogether, each member of the couple can receive up to $\$ 430$.

\section{Outcomes}

Primary outcome

The primary outcome is ART adherence. We will measure ARV levels in hair samples across all four time points. Additionally, assessments at each time point will measure engagement in HIV care using a comprehensive behavioural composite of engagement in HIV care. ${ }^{52}$

\section{Feasibility of app/intervention}

At T2 and T4, we will assess feasibility based on metrics in table 1 and metadata (eg, number of times the Action Plan was shared between partners, frequency of encouraging messages exchanged). We will code and tabulate these interactions to analyse dyadic HIV care engagement by, for example, the volume and sequence of activities planned. Participants can report glitches and other issues at any time through a reporting feature in the app or study website, or by contacting the study staff. All reports of issues will be tabulated.

We will monitor rates of recruitment and effort (eg, number of staff hours), number of screenings, proportion eligible and agreed to enrol, number of participants who withdraw after being randomised to condition and reason(s) for withdrawal and the number of participants who complete each time point. We will record the number of rescheduled, cancelled and missed visits to inform estimation of future staffing needs. Using call/time logs, we will record the frequency and mode of contact with participants, when, and for how long. During remote visits, staff will complete a checklist and take notes on study proceedings such as the procedures implemented, amount of time spent and participants' reactions. These 
Table 1 Metrics and thresholds to assess feasibility of the LetSync app

\begin{tabular}{|c|c|}
\hline Main feasibility outcomes & $\begin{array}{l}\text { Metrics } \\
\text { threshold }\end{array}$ \\
\hline Enrolment in both arms & $\begin{array}{l}\geq 70 \% \text { of } \\
\text { eligible } \\
\text { individuals } \\
\text { enrolled }\end{array}$ \\
\hline Retention in both arms at T2 & $\geq 75 \%$ retained \\
\hline Retention in both arms at T4 & $\geq 80 \%$ retained \\
\hline Number of app launches, log-ins & $\begin{array}{l}\text { Mean of once/ } \\
\text { week }\end{array}$ \\
\hline Number of minutes of app use & $\begin{array}{l}\text { Mean of } 10 \\
\mathrm{~min} / \text { week }\end{array}$ \\
\hline Use of the Our Action Plan feature & $\begin{array}{l}\geq 1 \text { Action Plan } \\
\text { generated/ } \\
\text { month }\end{array}$ \\
\hline Number of Action Plans created & $\begin{array}{l}\text { Mean of 1/ } \\
\text { month }\end{array}$ \\
\hline Communication between partners & $\begin{array}{l}\text { Mean of } 1 \\
\text { message/ } \\
\text { month }\end{array}$ \\
\hline Use of joint task feature & $\begin{array}{l}\text { Mean of } 1 \\
\text { joint task } \\
\text { completed/ } \\
\text { month }\end{array}$ \\
\hline Access of other LetSync features & $\begin{array}{l}\text { Mean of twice/ } \\
\text { month }\end{array}$ \\
\hline App opens following pop-up reminders & $\begin{array}{l}\text { Mean of } 50 \% \\
\text { of all pop-ups }\end{array}$ \\
\hline Number of app glitches & $\begin{array}{l}\text { Mean of } \leq 1 \\
\text { user-reported } \\
\text { glitch/week }\end{array}$ \\
\hline $\begin{array}{l}\text { Amount of time for Research Assistant to } \\
\text { field app questions }\end{array}$ & $\begin{array}{l}\text { Mean of } \\
\leq 1 \text { hour/week/ } \\
\text { participant }\end{array}$ \\
\hline
\end{tabular}

data will inform modifications to the intervention and protocols of a subsequent, full-scale efficacy trial.

We will compare HIV clinical outcomes and dyadic capacity measures between the two arms in exploratory analyses. We will evaluate feasibility and acceptability of LetSync V.2.0 in the waitlist-control arm and evaluate persistent use of LetSync V.1.0 over 14 months in the intervention arm.

\section{FEASIBILITY OF HAIR SAMPLE COLLECTION}

Feasibility of hair collection will be evaluated by: (1) the number of samples per participant received by the study, (2) the time difference between when remote hair samples were due versus when samples were received by the study and (3) rates of verifiable ARV results. Staff will document when hair collection kits were sent and received.
Table 2 Items and measures to assess acceptability of the LetSync app

\begin{tabular}{ll}
\hline Measure & Item \\
\hline App usability & $\begin{array}{l}\text { "I am satisfied with the app." } \\
\text { "I would want to use the app even if I } \\
\text { was not receiving study incentives." }\end{array}$ \\
$\begin{array}{l}\text { Security and } \\
\text { privacy }\end{array}$ & $\begin{array}{l}\text { "How secure did you feel about your } \\
\text { data when using the app?" }\end{array}$ \\
$\begin{array}{l}\text { Study procedures } \\
\text { and design }\end{array}$ & $\begin{array}{l}\text { "How helpful was the User's Guide } \\
\text { video you watched?" } \\
\text { "How satisfied were you with your } \\
\text { communication with the staff?" }\end{array}$ \\
$\begin{array}{l}\text { "Hemote hair } \\
\text { collection }\end{array}$ & $\begin{array}{l}\text { hair kits?" } \\
\text { "How easy or difficult was it to mail } \\
\text { your hair in?" } \\
\text { "How helpful was the demonstration } \\
\text { video?" }\end{array}$ \\
\hline $\begin{array}{l}\text { Remote study } \\
\text { participation }\end{array}$ & $\begin{array}{l}\text { participating in a remote research } \\
\text { project?" }\end{array}$ \\
\hline
\end{tabular}

\section{ACCEPTABILITY}

Acceptability will be evaluated via a measure of app usability ${ }^{53}$ and self-reported satisfaction with security and privacy of app use, study procedures and design and remote hair collection. During the exit interview, we will ask participants about what was convenient/easy versus inconvenient/difficult regarding remote study participation. The threshold for acceptability will be $80 \%$ of participants reporting being satisfied with the app content and delivery format. Table 2 contains examples of items used to capture each measure.

Throughout the intervention, we will contact participants in both arms monthly via text, call and/or email. We will check in about their experiences of using the app, along with troubleshooting app-related issues and sending in hair samples.

\section{DATA COLLECTION AND ANALYSIS}

\section{Quantitative data collection and analysis}

Assessments at baseline, 6 months, 8 months and 14 months will be administered online and will measure HIV care engagement using a comprehensive behavioural composite of engagement in HIV care $^{52}$; engagement and retention in care using the Index of Engagement in HIV Care (eg, "How well do you follow through on your HIV care when things in your life get tough?" $)^{54}$; and self-reported ART adherence (eg, "In the last 30 days, on how many days did you miss at least one dose of any of your medication?") ${ }^{55}$ and viral suppression (eg, "Was your last viral load detectable or undetectable?"). Guided by our conceptual framework (figure 1), ${ }^{22}$ we will measure dyadic capacity using the Dyadic Coping Inventory, ${ }^{56}$ Couple Health Support, Partner Support for HIV Treatment $^{57}$ and relationship factors using the Power 
Imbalance in Couples Scale ${ }^{58}$ and the Couple Sexual Satisfaction Scale (Conroy AA, Development and Validation of the Couple Sexual Satisfaction Scale for HIV and Sexual Health Research, Under Review). We will also assess individual-level factors as indicated by our conceptual framework, including the HIV Stigma Scale. ${ }^{59}$

Frequency tables will be generated for all clinical outcomes. One-way frequency tables will be generated for the number of rescheduled, cancelled and missed visits. Relative frequencies will be calculated for the number of participants enrolled in the study, those who were eligible in general and lost to follow-up. We will also tabulate and summarise acceptability outcomes in one-way frequency tables.

We will fit linear mixed models to continuous outcomes (eg, ARV levels in hair) and fit generalised linear mixed models to discrete (eg, viral suppression) and nonnormally distributed continuous outcomes (eg, selfreported ART adherence) to model outcome data. These analyses will include couple sero-status (sero-concordant HIV-positive vs sero-discordant) as a covariate as required by the stratified randomised design. ${ }^{6061}$ Following guidelines in the literature ${ }^{62}{ }^{63}$ and from $\mathrm{NIH},{ }^{64}$ hypothesis testing will de-emphasised. Instead, we will perform these analyses to ensure that all measures and procedures are well established to perform a subsequent efficacy trial.

\section{QUALITATIVE DATA COLLECTION AND ANALYSIS}

At T4, staff will conduct remote exit interviews with all participants. Exit interviews will explore participants' experiences with the study protocol and procedures. Interviews will be audio-recorded and professionally transcribed.

We will read all individual transcripts and develop a codebook based on the interview guides, our theoretical framework and emergent themes. To establish intercoder agreement, a primary coder will apply codes based to a subset of transcripts to test and revise the codebook. A secondary analyst will apply the revised set of codes on a random subset of transcripts. Discrepancies in coding will be discussed by the team until an agreement is reached.

\section{POWER ANALYSES}

We estimated minimum detectable effect sizes (MDEs) for the assessments of feasibility and acceptability proposed to address the pilot RCT. We anticipate 80 couples $(40$ seroconcordant-positive and 40 serodiscordant per condition) at the beginning of the study and 64 couples at T4 following $20 \%$ estimated attrition. The effective sample size (ESS) will depend on the unit of analysis (couple vs individual), which participants are included in the analysis and when the outcome is measured. For instance, the enrolment proportion to assess feasibility is a couple-level variable measured at the outset of the study. Assuming $\alpha=0.05$, power $=0.80$ and $70 \%$ enrolment for 114 couples contacted to yield 80 couples ( $70 \%$ of 114 ), the width of the CI for single enrolment proportions is $19 \%$ (standardised distance to the limit: 0.20 ). In contrast, acceptability scores will be measured at the individual level at the study end point among participants in each condition.

We also performed power analyses for proposed outcome analyses in order to supply additional information. For individual-level outcomes, the ESS will depend on the degree of within couple correlation of responses, $\rho$, within couples. We set $\rho$ based on prior dyadic research in which the average within-couple correlation of virological control measurements was $\rho=0.23$. Accordingly, we lowered the ESS inputted for the power analyses to be $\mathrm{ESS}=\mathrm{N} / \mathrm{DEFF}$, where $\mathrm{N}$ is the end point sample size and DEFF is the design effect or variance inflation attributable to using correlated data. DEFF is computed as $1+(\mathrm{M}-1) \times \rho$, where $\mathrm{M}$ is the number of participants per dyad (ie, 2). Therefore, $\mathrm{DEFF}=1+(2-1) \times 0.23=1.23$, so $\mathrm{ESS}=80 \times 0.80=64 / 1.23=52$. Under these assumptions, distance from the observed mean to the confidence limit is estimated to be 0.28 . For longitudinal analyses to evaluate ART adherence, outcomes will be measured at the individual level at every time point among HIV + participants in both arms. An $80 \%$ retention rate means $20 \times 0.80=16$ seroconcordant-positive couples yielding $32 \mathrm{HIV}+$ participants, where ESS=32/1.23=26 plus $20 \times 0.80=16$ serodiscordant couples yielding 16 HIV + participants for a total end point study sample of 42 per arm. Assuming $\alpha=0.05$, power $=0.80$ and four time points with $\mathrm{r}=0.30$ correlation between repeated measures (in Dr Johnson's study, the average within-subject r's for ART adherence and viral suppression were 0.24 and 0.28 , respectively), the minimum detectable standardised mean differences for continuous outcomes is 0.421 . For binary outcomes, using the same inputs as above plus small, medium and large base rates of $10 \%, 30 \%$ and $50 \%$, respectively, raw proportion differences range from $16.1 \%$ to $20.5 \%$ (standardised difference $=0.422-0.429)$. H1-H3 will be directly tested by contrasts derived from the longitudinal analytic models. For H1 and H3, we estimated the MDEs of those contrasts by reassessing the power of the longitudinal analyses with only two time points. The resulting effect sizes ranged from 0.493 to 0.503 , which are medium standardised effects. For H2, MDEs for a non-zero longitudinal change in a group mean or proportion range from 0.407 to 0.470 , which are small to medium standardised effects. As noted previously, hypothesis testing will be de-emphasised in this pilot feasibility and acceptability study.

\section{DISCUSSION}

This paper describes the protocol for a randomised waitlist-controlled pilot of a dyadic app intervention, LetSync, focused on black sexual-minority couples living with HIV. Barriers to HIV care for black MSM are multilevel, often at the social (eg, HIV stigma) and structural (eg, transportation) levels, while extant interventions target barriers at the individual level. LetSync addresses 
this gap by targeting, at the dyadic level, black MSM couple dynamics, emphasising the roles of dyadic coordination and joint problem-solving in improving HIV care engagement.

Although black MSM-centred mHealth interventions exist in general, ${ }^{32}{ }^{65}$ there is a paucity of couples-based mHealth studies for this population despite the demonstrated power of dyadic coordination in care, and couples facing many unique barriers to care and treatment.

A search in the literature yielded only one couplesbased mHealth study for black MSM. In 2010, an existing evidence-based intervention originally developed for heterosexual couples was adapted for black MSM to reduce sexually transmitted infections (STIs; including HIV and other STIs) and drug use outcomes. This adaptation was recently piloted with 34 MSM dyads with promising results. ${ }^{66}{ }^{67}$ Of the seven couple-based HIV studies that have been conducted since the start of the HIV epidemic, only three have included MSM in general, and none included black MSM ${ }^{66}$

Our study addresses the lack of couples-based interventions for black MSM in several innovative ways. It seeks to harness couples' resilience and ability to synchronise problem-solving approaches, both of which are likely to impact dyadic coordination and joint problem-solvingthus improving HIV care engagement. ${ }^{14}$ It is also informed by our theoretical framework, the Framework of Dyadic HIV Care Engagement, which is formulated by preliminary and existing research. Rather than focus on single users' experiences and outcomes, as is the case for most traditional mHealth designs (including HIV prevention), ${ }^{34} 68$ the design of LetSync targets the dyad where each user's outcomes are dependent on the joint, collaborative, synchronised behaviours of both users. The dyadic level is often missing in multilevel HIV prevention efforts, but retention in care and ART adherence often occur in the dyadic context for black sexual-minority couples. ${ }^{14}$ Lastly, our study is the first of its kind to include the use of remote hair collection to measure ART adherence. Hair concentrations of ARVs are stronger predictors of virological suppression than self-reported adherence or plasma ARV levels in large cohort studies of patients with HIV. ${ }^{69}$ Self-collection of hair samples at home reduces travel time and expenses, and assessing our primary outcome via remote collection of hair is congruent with the mobile nature of the intervention.

There are several challenges to this study. Suboptimal app engagement poses a challenge in mHealth data collection. To optimise app engagement, we will programme pop-up reminders to appear on a weekly basis if the app has not been opened. We will assess the feasibility and acceptability of this feature during exit interviews. To minimise participant attrition, which is intrinsic to longitudinal designs, we will collect at least three methods of personal contact such as social media handles and additional phone numbers. We will also maintain regular contact with participants by sending reminders about virtual check-ins and sending in hair samples and asking about any app-related issues. Lastly, addressing break-ups is necessary as our study involves couples. If break-up occurs between screening and randomisation, the couple will become ineligible and referrals for support will be offered to both participants. If break-up occurs after randomisation, participants may still take part in the remaining data collection time points as scheduled, and the breakup will be noted in the retention and tracking study databases.

This paper documents the protocol for the LetSync study, which was designed to help couples work together to improve HIV-related outcomes. While the number of HIV-centred mHealth interventions have proliferated in recent years, very few exist that focus on black MSM in couples. mHealth for dyadic HIV care engagement holds promise in being cost-efficient and transcending common barriers to intervention and care, which our study aims to demonstrate. Findings from the proposed research are needed for a subsequent large-scale, RCT to test the efficacy of LetSync in improving HIV care and treatment outcomes among black MSM. These findings may inform future studies and protocols for other chronic conditions where the dyad is an important unit of intervention.

\section{Ethics and dissemination}

This study has been approved by the University of California (UCSF) Institutional Review Board (\#15-18042). Informed consent will be obtained electronically (eg, via Qualtrics). Participants will be informed that their participation in the study is voluntary and that they may decline to participate for any reason without any negative consequences. Referrals for emotional support and mental health will be available.

Results of the pilot RCT will be disseminated through peer-reviewed publications, conferences and presentations and reports to participants and stakeholders. We will also hold Town Halls with the UCSF Center for AIDS Prevention Studies and symposia with community-based organisations that serve people living with HIV.

\section{Acknowledgements The authors would like to thank Sage Bionetworks for granting LetSync the Digital Health Catalyst Award.}

Contributors JT designed the study, obtained funding, provided leadership in the execution of the study and contributed to revising the manuscript. TBN, LP, PS, EA and SK contributed to study conception. TBN and LP also contributed to trial design. The paper was drafted by HCK, and all authors, including DJB and RWW, read and approved the final manuscript.

Funding This research was supported by a grant from the National Institute of Mental Health R01MH118967 (JT).

Disclaimer The contents of this publication are solely the responsibility of the authors and do not represent the official views of the National Institutes of Health.

\section{Competing interests None declared.}

Patient and public involvement Patients and/or the public were involved in the design, or conduct, or reporting, or dissemination plans of this research. Refer to the 'Methods and analysis' section for further details.

Patient consent for publication Not required.

Provenance and peer review Not commissioned; peer reviewed for ethical and funding approval prior to submission. 
Open access This is an open access article distributed in accordance with the Creative Commons Attribution Non Commercial (CC BY-NC 4.0) license, which permits others to distribute, remix, adapt, build upon this work non-commercially, and license their derivative works on different terms, provided the original work is properly cited, appropriate credit is given, any changes made indicated, and the use is non-commercial. See: http://creativecommons.org/licenses/by-nc/4.0/.

\section{ORCID iDs}

Hyunjin Cindy Kim http://orcid.org/0000-0002-3322-7215

Parya Saberi http://orcid.org/0000-0002-3793-5112

\section{REFERENCES}

1 Centers for Disease Control and Prevention. Estimated HIV incidence and prevalence in the United States, 2015-2019, 2021. Available: https://www.cdc.gov/hiv/pdf/library/reports/surveillance/cdc-hivsurveillance-supplemental-report-vol-26-1.pdf [Accessed 16 Mar 2021].

2 HIV and African American gay and bisexual men. centers for disease control and prevention, 2020. Available: https://www.cdc.gov/hiv/ group/msm/bmsm.html [Accessed 13 Apr 2021].

3 Singh S, Mitsch A, Wu B. HIV care outcomes among men who have sex with men with diagnosed HIV infection - United States, 2015. MMWR Morb Mortal Wkly Rep 2017:66:969-74.

4 Centers for Disease Control and Prevention. Estimated HIV incidence and prevalence in the United States, 2014-2018, 2020. Available: https://www.cdc.gov/hiv/pdf/library/reports/surveillance/cdc-hivsurveillance-supplemental-report-vol-25-1.pdf [Accessed 8 Jun 2021].

5 Hall HI, Holtgrave DR, Tang T, et al. HIV transmission in the United States: considerations of viral load, risk behavior, and health disparities. AIDS Behav 2013;17:1632-6.

6 McMahon JM, Braksmajer A, Zhang C, et al. Syndemic factors associated with adherence to antiretroviral therapy among HIVpositive adult heterosexual men. AIDS Res Ther 2019;16:32.

72016 CROI press release: lifetime risk of HIV diagnosis., 2016. Available: https://www.cdc.gov/nchhstp/newsroom/2016/croi-pressrelease-risk.html [Accessed 16 Mar 2021].

8 Hess KL, Hu X, Lansky A, et al. Lifetime risk of a diagnosis of HIV infection in the United States. Ann Epidemiol 2017;27:238-43.

9 Eaton LA, Matthews DD, Bukowski LA, et al. Elevated HIV prevalence and correlates of PrEP use among a community sample of black men who have sex with men. J Acquir Immune Defic Syndr 2018;79:339-46.

10 Koblin BA, Mayer KH, Eshleman SH, et al. Correlates of HIV acquisition in a cohort of black men who have sex with men in the United States: HIV prevention trials network (HPTN) 061. PLoS One 2013;8:e70413.

11 Okafor CN, Hucks-Ortiz C, Hightow-Weidman LB, et al. Brief report: associations between self-reported substance use behaviors and PreP acceptance and adherence among black MSM in the HPTN 073 study. J Acquir Immune Defic Syndr 2020;85:23-9.

12 Umberson D, Montez JK. Social relationships and health: a flashpoint for health policy. J Health Soc Behav 2010;51(Suppl):S54-66.

13 Uchino BN, Bowen K, Kent de Grey R, et al. Social support and physical health: models, mechanisms, and opportunities. In: Fisher EB, Cameron LD, Christensen AJ, eds. Principles and concepts of behavioral medicine: a global Handbook. Springer, 2018: 341-72.

14 Goldenberg T, Clarke D, Stephenson R. "Working together to reach a goal": MSM's perceptions of dyadic HIV care for same-sex male couples. J Acquir Immune Defic Syndr 2013;64(Suppl 1):S52-61.

15 Goldenberg T, Stephenson R. "The more support you have the better": partner support and dyadic HIV care across the continuum for gay and bisexual men. J Acquir Immune Defic Syndr 2015;69(Suppl 1):S73-9.

16 Sullivan PS, Peterson J, Rosenberg ES, et al. Understanding racial HIV/STI disparities in black and white men who have sex with men: a multilevel approach. PLoS One 2014;9:e90514.

17 Tan JY, Pollack L, Rebchook G, et al. The role of the primary romantic relationship in HIV care engagement outcomes among young HIV-positive black men who have sex with men. AIDS Behav 2018;22:774-90.

18 Tan JY, Campbell CK, Tabrisky AP, et al. A conceptual model of Dyadic coordination in HIV care engagement among couples of black men who have sex with men: a qualitative Dyadic analysis. AIDS Behav 2018;22:2584-92.

19 Kayser K. Enhancing dyadic coping during a time of crisis: an intervention with breast cancer patients and their partners. In: Revenson TA, Kayser K, Bodenmann G, eds. Couples coping with stress: emerging perspectives on Dyadic coping. American Psychological Association, 2005: 175-94.

20 Widmer K, Cina A, Charvoz L. A model dyadic coping intervention. In: Revenson TA, Kayser K, Bodenmann G, eds. Couples coping with stress: emerging perspectives on Dyadic coping. American Psychological Association, 2005: 159-74.

21 Gamarel KE, Revenson TA. Dyadic adaptation to chronic illness: the importance of considering context in understanding couples' resilience. In: Skerrett K, Fergus K, eds. Couple resilience: emerging perspectives. Springer Science + Business Media, 2015: 83-105.

22 Tan JY, Campbell CK, Conroy AA, et al. Couple-Level dynamics and multilevel challenges among black men who have sex with men: a framework of Dyadic HIV care. AIDS Patient Care STDS 2018;32:459-67.

23 Pew Research Center: Internet, Science \& Tech. Demographics of mobile device ownership and adoption in the United States, 2021. Available: https://www.pewresearch.org/internet/fact-sheet/mobile/ [Accessed 14 Apr 2021].

24 Ybarra ML, Prescott TL, Phillips GL, et al. Pilot RCT results of an mHealth HIV prevention program for sexual minority male adolescents. Pediatrics 2017;140.

25 Balán IC, Lopez-Rios J, Nayak S, et al. SMARTtest: a smartphone APP to facilitate HIV and syphilis self- and Partner-Testing, interpretation of results, and linkage to care. AIDS Behav 2020;24:1560-73.

26 Hightow-Weidman L, Muessig K, Knudtson K, et al. A Gamified smartphone APP to support engagement in care and medication adherence for HIV-positive young men who have sex with men (AllyQuest): development and pilot study. JMIR Public Health Surveill 2018;4:e8923.

27 Hightow-Weidman LB, Pike E, Fowler B, et al. HealthMpowerment. org: feasibility and acceptability of delivering an Internet intervention to young black men who have sex with men. AIDS Care 2012;24:910-20.

28 Hightow-Weidman LB, Smith JC, Valera E, et al. Keeping them in "STYLE": finding, linking, and retaining young HIV-positive black and Latino men who have sex with men in care. AIDS Patient Care STDS 2011;25:37-45.

29 Muessig KE, Pike EC, Fowler B, et al. Putting prevention in their pockets: developing mobile phone-based HIV interventions for black men who have sex with men. AIDS Patient Care STDS 2013;27:211-22.

30 Khosropour CM, Lake JG, Sullivan PS. Are MSM willing to SMS for HIV prevention? J Health Commun 2014;19:57-66.

31 Khosropour CM, Sullivan PS. Predictors of retention in an online follow-up study of men who have sex with men. J Med Internet Res 2011;13:e47.

32 Khosropour CM, Johnson BA, Ricca AV, et al. Enhancing retention of an Internet-based cohort study of men who have sex with men (MSM) via text messaging: randomized controlled trial. J Med Internet Res 2013;15:e194.

33 Sullivan PS, Grey JA, Simon Rosser BR. Emerging technologies for HIV prevention for MSM: what we have learned, and ways forward. J Acquir Immune Defic Syndr 2013;63(Suppl 1):S102-7.

34 Muessig KE, LeGrand S, Horvath KJ, et al. Recent mobile health interventions to support medication adherence among HIV-positive MSM. Curr Opin HIV AIDS 2017;12:432-41.

35 Pasipanodya EC, Montoya JL, Watson CW-M, et al. Tailoring a mobile health text-messaging intervention to promote antiretroviral therapy adherence among African Americans: a qualitative study. PLoS One 2020;15:e0233217.

36 Tan JYR, Nguyen TT, Tabrisky A, et al. Mobile technology for healthy aging among older HIV-positive black men who have sex with men: qualitative study. JMIR Aging 2018;1:e11723.

37 Irvin $\mathrm{R}$, Wilton $\mathrm{L}$, Scott $\mathrm{H}$, et al. A study of perceived racial discrimination in black men who have sex with men (MSM) and its association with healthcare utilization and HIV testing. AIDS Behav 2014;18:1272-8

38 Hoots BE, Finlayson TJ, Wejnert C, et al. Updated data on linkage to human immunodeficiency virus care and antiretroviral treatment among men who have sex with Men-20 cities, United States. J Infect Dis 2017;216:808-12.

39 Sullivan PS, Knox J, Jones J, et al. Understanding disparities in viral suppression among black MSM living with HIV in Atlanta Georgia. J Int AIDS Soc 2021;24:e25689.

40 Palar K, Laraia B, Tsai AC, et al. Food insecurity is associated with HIV, sexually transmitted infections and drug use among men in the United States. AIDS 2016;30:1457-65.

41 Creasy SL, Henderson ER, Bukowski LA, et al. Hiv testing and art adherence among unstably housed black men who have sex with men in the United States. AIDS Behav 2019;23:3044-51. 
42 Quinn K, Dickson-Gomez J, Zarwell M, et al. "A Gay Man and a Doctor are Just like, a Recipe for Destruction": How Racism and Homonegativity in Healthcare Settings Influence PrEP Uptake Among Young Black MSM. AIDS Behav 2019;23:1951-63.

43 Maguire M. Methods to support human-centred design. Int J Hum Comput Stud 2001;55:587-634.

44 Johnson MO, Dilworth SE, Taylor JM, et al. Primary relationships, HIV treatment adherence, and virologic control. AIDS Behav 2012;16:1511-21.

45 Amico KR, Fisher WA, Cornman DH, et al. Visual analog scale of ART adherence: association with 3-day self-report and adherence barriers. J Acquir Immune Defic Syndr 2006;42:455-9.

46 Tan J, Conroy A, Lee I, et al. Leveraging power in intimate partner relationships: a power bases perspective. In: Agnew CR, Harman JJ, eds. Power in close relationships. Cambridge University Press, 2017.

47 Sheon N, Lee S-H. Sero-skeptics: discussions between test counselors and their clients about sexual partner HIV status disclosure. AIDS Care 2009;21:133-9.

48 D'Zurilla TJ, Nezu AM. Problem-solving therapy. In: Dobson KS, ed Handbook of cognitive-behavioral therapies. 3rd ed. Guilford Press, 2010: 197-225.

49 Anguera JA, Gunning FM, Areán PA. Improving late life depression and cognitive control through the use of therapeutic video game technology: a proof-of-concept randomized trial. Depress Anxiety 2017;34:508-17.

50 Hair collection instructions. The RxPix study. Available: https://rxpix ucsf.edu/hair-collection-instructions [Accessed 8 Jun 2021].

51 Saberi P, Ming K, Legnitto D, et al. Novel methods to estimate antiretroviral adherence: protocol for a longitudinal study. Patient Prefer Adherence 2018;12:1033-42.

52 Saberi $\mathrm{P}$, Johnson MO. Moving toward a novel and comprehensive behavioral composite of engagement in HIV care. AIDS Care 2015;27:660-4.

53 Brooke J. System usability scale (SUS), 1986. Available: http://www. usability.gov/how-to-and-tools/methods/system-usability-scale.html [Accessed 11 Jun 2021].

54 Johnson MO, Neilands TB, Koester KA, et al. Detecting disengagement from HIV care before it is too late: development and preliminary validation of a novel index of engagement in HIV care. $J$ Acquir Immune Defic Syndr 2019;81:145-52.

55 Wilson IB, Lee Y, Michaud J, et al. Validation of a new ThreeItem self-report measure for medication adherence. AIDS Behav 2016;20:2700-8.
56 Bodenmann G. Dyadisches Coping Inventar: Testmanual [Dyadic Coping Inventory: Test Manual], 2008.

57 PI: Johnson MO. Title of grant: a couples-based approach to improving engagement in HIV care. University of California, San Francisco. National Institute of Nursing Research, 2006: (\$602,288):5R01NR010187

58 Neilands TB, Dworkin SL, Chakravarty D, et al. Development and validation of the power imbalance in couples scale. Arch Sex Behav 2019;48:763-79.

59 Berger BE, Ferrans CE, Lashley FR. Measuring stigma in people with HIV: psychometric assessment of the HIV stigma scale. Res Nurs Health 2001;24:518-29.

60 Altman DG, Doré CJ. Randomisation and baseline comparisons in clinical trials. Lancet 1990;335:149-53.

61 Kernan WN, Viscoli CM, Makuch RW, et al. Stratified randomization for clinical trials. J Clin Epidemiol 1999;52:19-26.

62 Kraemer HC, Mintz J, Noda A, et al. Caution regarding the use of pilot studies to guide power calculations for study proposals. Arch Gen Psychiatry 2006;63:484-9.

63 Leon AC, Davis LL, Kraemer HC. The role and interpretation of pilot studies in clinical research. J Psychiatr Res 2011;45:626-9.

64 National Center for Complementary and Integrative Health. Pilot studies: common uses and misuses. Available: https://www.nccih. nih.gov/grants/pilot-studies-common-uses-and-misuses [Accessed 2 Jun 2021].

65 Rouffiac A-E, Whiteley L, Brown L, et al. A mobile intervention to improve uptake of pre-exposure prophylaxis for southern black men who have sex with men: protocol for intervention development and pilot randomized controlled trial. JMIR Res Protoc 2020;9:e15781.

66 El-Bassel N, Gilbert L, Witte S, et al. Couple-based HIV prevention in the United States: advantages, gaps, and future directions. J Acquir Immune Defic Syndr 2010;55(Suppl 2):S98-101.

67 Wu E, El-Bassel N, Donald McVinney L, et al. Adaptation of a couple-based HIV intervention for Methamphetamine-Involved African American men who have sex with men. Open AIDS J 2010;4:123-31.

68 Mitchell JW. The use of technology to advance HIV prevention for couples. Curr HIVIAIDS Rep 2015;12:516-22.

69 Baxi SM, Liu A, Bacchetti P, et al. Comparing the novel method of assessing PrEP adherence/exposure using hair samples to other pharmacologic and traditional measures. J Acquir Immune Defic Syndr 2015;68:13-20. 